\title{
A Hesitant Fuzzy Envelope Based Expert System in Human Decision Making
}

\author{
Panjkaj Srivastava ${ }^{1} \&$ Rajkrishna Mondal ${ }^{2}$ \\ Department of Mathematics \\ Motilal Nehru National Institute of Technology Allahabad, Uttar Pradesh, India \\ Emails:12ankajs@mnnit.ac.in \& ${ }^{2}$ rajkrishna@mnnit.ac.in
}

\begin{abstract}
Naturally, individual decision style is qualitative rather than quantitative settings. In nature, the human way of thinking is uncertain and fuzziness that demands the use of the linguistic approach of problems related to the decision. The group decision making process is highly affected by hesitant situations among the members for clarity-based decisions. In order to remove the hesitant situations, the proposed Hesitant Fuzzy Envelope expert system provides the group decision making processes with more realistic output in envelope form rather than CRISP one.In this study, we shall discuss a linguisticbased expert system that will help to make more realistic decisions in a hesitant situation by using Hesitant Fuzzy Envelope technique.
\end{abstract}

Keywords: Expert systems, Human decision making, Hesitant Fuzzy envelope, Soft computing

\section{Introduction}

In human beings, decision making, is a natural process, based on mental and reasoning processes in the uncertain, imprecise, and vague environment [5]. To model this type of decision-making problem, we need linguistic information that comes from experts to express their knowledge in qualitative value.

The linguistic model has successfully provided for managing the complexity of real-world problems. Most of the linguistic model make a decision based on a single linguistic term. But in a high degree of uncertainty depends on a linguistic context where the experts have hesitated among the different linguistic terms.

There have different types of literature to provide linguistic context rather than a single linguistic term. Merging different types of linguistic terms into a single word, Ma et al. [6] increase the flexibility of the linguistic model. By using logical connectives, Tang and Zheng [9] build a linguistic model to manage linguistic expressions. Rodriguez et al. [7] has introduced the Hesitant Fuzzy Linguistic term set (HFLTS) to improve the importance of the linguistic decision-making model. Most of the Hesitant Fuzzy Linguistic model operates with a symbolic model, and the final output gives CRISP values, so initial fuzzy representation has lost in outcomes. So, the aim of this present study is that developed an expert system using the Fuzzy Envelop concept in HFLTS where the results in this decision-making model come in the form of Fuzzy membership function.

The present article is structured as follows: Section 2 discusses some preliminary concepts. Section 3 discusses about Hesitant Fuzzy Envelop. Section 4 introduces an expert system using Hesitant Fuzzy Envelop concept. Section 5 deals with Validation and Advantage of the proposed expert system. Section 6: Draw a proper conclusion. 


\section{Preliminaries}

\subsection{Linguistic Variable [11]}

Definition 1: A linguistic variable is defined by a quintuple $(H, T(H), U, G, M)$ in which $H$ is name of the variable, $T(H)$ is the term set of $H, U$ is the universe of discourse, $G$ is a syntactic rule which generates $T(H), M$ is a semantic rule associated with each linguistic value.

It is necessary to choose a linguistic descriptor to deal with a linguistic variable. For selecting linguistic descriptor, there have various kind of approaches. But in our cases,

Let $S=\left\{s_{0}, s_{1}, s_{2}, \cdots, s_{\# S}\right\}$ be a set of linguistic terms satisfied

1. $s_{i} \leq s_{j}$ iff $i \leq j$

2. Negation $\left(s_{i}\right)=s_{\# S-i}$

3. $\max \left(s_{i}, s_{j}\right)=s_{i}$ and $\min \left(s_{i}, s_{j}\right)=s_{j}$ if $i \geq j$.

\subsection{A Context-free grammar [1] [2]}

A context-free grammar $(G)$ is used for generating linguistic expressions utilizing the set of linguistic terms.

Definition 2: $G$, mainly defined in 4-tuple $\left(V_{N}, V_{T}, I, P\right)$ where $V_{N}$ : Non-terminal symbols,

$V_{T}$ : Terminal symbols, $I$ : Starting symbols, : Production Rules

$\mathrm{P}=\{$ (Primary Term) or (Unary Relation) (Primary Term)

or (Binary Relation) (Primary Term) (Conjunction) (Primary Term) $\}$

$\left(\right.$ Primary Term) $=s_{i} \in S$

$($ Unary Relation $)=$ Lower Than or Greater Than

$($ Binary Relation $)=$ Between

$($ Conjunction $)=$ And

\subsection{Hesitant Fuzzy Linguistic Term Set (HFLTS) [7]}

Definition 3: Let $S=\left\{s_{0}, s_{1}, \cdots, s_{\# S}\right\}$ be a linguistic term set, an HFLTS, $H_{S}$, is an ordered finite subset of the consecutive linguistic terms of $S$.

$$
\therefore H_{S}=\left\{s_{i}, s_{i+1}, \cdots, s_{j} \mid s_{i}, s_{i+1}, \cdots, s_{j} \in S\right\} \text {. }
$$

Transformation Function: The function $E_{G}$ transform a linguistic expression $S_{l l}$ which is generated by a context-free grammar $G$ into an HFLTS $H_{S}$.

$$
\therefore E_{G}: S_{l l} \rightarrow H_{S} \text {. }
$$

\subsection{ORness and ANDness operators [10]}

Let $A=\left[a_{1}, a_{2}, \cdots, a_{n}\right]$ be a vector of $n$ elements and $B=\left[b_{1}, b_{2}, \cdots, b_{n}\right]$ where $b_{j}$ is the $j$ th largest element of $A . W=\left[w_{1}, w_{2}, \cdots, w_{n}\right]^{T}$ be the associated weight vector satisfying $w_{i} \in[0,1], i=1,2, \cdots, n$ and $\sum_{i=1}^{n} w_{i}=1$. Then ordered weighting average (OWA) operator on $A$ is defined by

$$
O W A_{W}(A)=B . W
$$

Definition 4: The ORness operator corresponding to the weighting vector $W$ is also an OWA operator and defined as

$$
\operatorname{ORness}(W)=\frac{1}{n-1} \sum_{i=1}^{n} w_{i}(n-i)
$$

Where $0 \leq \operatorname{ORness}(W) \leq 1$, and $\operatorname{ANDness}(W)$ is defined by $\operatorname{ANDness}(W)=1-\operatorname{ORness}(W)$.

Optimistic OWA operator or OR like OWA operator are those whose ORness $(W)>0.5$ and pessimistic OWA or AND like OWA operator are those whose $\operatorname{ANDness}(W)<0.5$. 


\section{Hesitant Fuzzy Envelope}

Let $S=\left\{s_{0}, s_{1}, s_{2}, \cdots, s_{\# S}\right\}$ be a set of linguistic terms and $H_{S}$ be an HFLTS on $S$ such that

$$
H_{S}=\left\{s_{i}, s_{i+1}, \cdots, s_{j} \mid s_{i}, \cdots, s_{j} \in S\right\} .
$$

Also, let each $s_{i}$ in $S$ is defined in the form of a triangular membership function $T(a, b, c)[11]$

Hesitant Fuzzy Envelope calculation is performed into three steps:

\subsection{Finding the elements}

Let $A^{k}=T\left(a_{L}^{k}, a_{M}^{k}, a_{R}^{k}\right), k=0,1, \cdots, \# S$ be membership function of $s_{k} \in S$.

But according to fuzzy partition [8] $a_{R}^{k-1}=a_{M}^{k}=a_{L}^{k+1}$

So, the points of all the linguistic terms in $H_{S}$ is

$$
T\left(H_{S}\right)=\left\{a_{L}^{i}, a_{M}^{i}, a_{M}^{i+1}, a_{M}^{i+2}, \cdots, a_{M}^{j}, a_{R}^{j}\right\}
$$

\subsection{Compute the parameters of the membership function for fuzzy envelope}

The fuzzy envelope of $H_{S}$ can be represented by the trapezoidal membership function $T(a, b, c, d)$ which can calculate from the points of $T\left(H_{S}\right)$ where

$$
\begin{aligned}
& a=\min \left\{a_{L}^{i}, a_{M}^{i}, a_{M}^{i+1}, a_{M}^{i+2}, \cdots, a_{M}^{j}, a_{R}^{j}\right\} \\
& d=\max \left\{a_{L}^{i}, a_{M}^{i}, a_{M}^{i+1}, a_{M}^{i+2}, \cdots, a_{M}^{j}, a_{R}^{j}\right\}
\end{aligned}
$$

For computing $b$ and $c$, there arise two cases:

a) If $i+j=$ Odd

$$
\begin{aligned}
& \text { i. If } i+1=j \\
& b=a_{M}^{i} \text { and } c=a_{M}^{i+1}
\end{aligned}
$$

ii. If $i+1<j$

b) If $i+j=$ Even

$$
\begin{aligned}
& b=O W A_{W^{s}}\left(a_{M}^{i}, a_{M}^{i+1}, a_{M}^{i+2}, \cdots, a_{M}^{\frac{i+j-1}{2}}\right) \\
& c=O W A_{W^{t}}\left(a_{M}^{j}, a_{M}^{j-1}, a_{M}^{j-2}, \cdots, a_{M}^{\frac{i+j+1}{2}}\right)
\end{aligned}
$$

$$
\begin{aligned}
& b=O W A_{W^{s}}\left(a_{M}^{i}, a_{M}^{i+1}, a_{M}^{i+2}, \cdots, a_{M}^{\frac{i+j}{2}}\right) \\
& c=O W A_{W^{t}}\left(a_{M}^{j}, a_{M}^{j-1}, a_{M}^{j-2}, \cdots, a_{M}^{\frac{i+j}{2}}\right)
\end{aligned}
$$

Where the weighting vector $W^{s}$ and $W^{t}$ are computed from the next step.

\subsection{Compute the weighting vector $W^{s}$ and $W^{t}$}

Let $\alpha \in[0,1]$ be a parameter then through the formula we have calculated $W^{s}$ and $W^{t}$ vectors:

$$
W^{S}=\left[w_{1}^{S}, w_{2}^{S}, w_{3}^{S}, \cdots, w_{n}^{S}\right]^{T}, \text { where } w_{1}^{S}=\alpha^{n-1} ; w_{i}^{S}=(1-\alpha) \alpha^{n-i}, i=2,3, \cdots, n
$$

and

$$
W^{t}=\left[w_{1}^{t}, w_{2}^{t}, w_{3}^{t}, \cdots, w_{n}^{t}\right]^{T}, \text { where } w_{1}^{t}=\alpha ; w_{i}^{t}=\alpha(1-\alpha)^{i-1}, i=2,3, \cdots, n .
$$




\section{Expert System}

Let in an expert system there have $n$ alternatives $A=\left\{a_{1}, a_{2}, \cdots, a_{n}\right\}$ and $m$ criteria $C=\left\{c_{1}, c_{2}, \cdots, c_{m}\right\}$. With the help of expert panels, for each alternative $a_{i}$ corresponding to each criterion $c_{j}$, we have built Hesitant Fuzzy Linguistic Matrix HFLM $=\left[H_{S}^{i j}\right]_{n \times m}$ of order $n \times m$ from a linguistic term set $S=\left\{s_{0}, s_{1}, s_{2}, \cdots, s_{\# S}\right\}$.

Now the expert system is executed through the following steps:

\subsection{Convert HFLM elements to the Fuzzy Envelope}

In this step, we have converted each $\operatorname{HFLTS} H_{S}^{i j} ; i=1,2, \cdots, n$ and $j=1,2, \cdots, m$ into a fuzzy envelope through the steps, discussed in section 3.

Let fuzzy envelope of $H_{S}^{i j}=T\left(a^{i j}, b^{i j}, c^{i j}, d^{i j}\right)$

\subsection{Averaging the fuzzy envelopes for each alternative}

From the previous steps, we have got $m$ fuzzy envelopes (corresponding to each criteria) for each alternative $a_{i}$.

Now we have averaging these $m$ fuzzy envelopes for each $a_{i}$ using the formula.

$$
\begin{aligned}
T\left(a_{i}\right) & =T\left(\frac{1}{m} \sum_{j=1}^{m}\left(a^{i j}, b^{i j}, c^{i j}, d^{i j}\right)\right) \\
& =T\left(a^{i}, b^{i}, c^{i}, d^{i}\right), i=1,2 \cdots, n
\end{aligned}
$$

\subsection{Preference Relation between alternatives}

In this step, we have built the preference relation [4] matrix $P=\left[p_{i j}\right]_{n \times n}$ by distance measuring between each pair of alternatives $\left(a_{i}, a_{j}\right), i, j=1,2 \cdots, n$. Distance measuring between two fuzzy envelopes [3] are as follows

$$
\begin{aligned}
p_{i j} & =d\left(T\left(a_{i}\right), T\left(a_{j}\right)\right) \\
& =\frac{1}{4}\left(\left|a^{i}-a^{j}\right|^{p}+\left|b^{i}-b^{j}\right|^{p}+\left|c^{i}-c^{j}\right|^{p}+\left|d^{i}-d^{j}\right|^{p}\right)^{\frac{1}{p}}, \text { if } 1 \leq p<\infty, i \neq j
\end{aligned}
$$

\subsection{Calculate non-dominance degree}

The non-dominance degree [4] $N D D_{i}$ for each alternative $a_{i}, i=1,2, \cdots, n$ is calculated by

$$
N D D_{i}=\min \left\{1-p_{j i} \mid j \neq i, j=1,2, \cdots, n\right\}
$$

\subsection{Finding the best alternatives}

According to the maximum value of the non-dominance degree $N D D_{i}$ for alternative $a_{i}$ calculated the best alternative.

So,

$$
A^{\text {Best }}=\left\{a_{i} \mid N D D_{i}=\operatorname{Max}_{a_{j} \in A}\left(N D D_{j}\right)\right\}
$$

\section{Validation and Advantage}

\subsection{Validation}

To validate the proposed expert system, we have taken the example which has given in [7]. Let there have three alternatives $X=\left\{x_{1}, x_{2}, x_{3}\right\}$ and three criteria 


$$
C=\left\{c_{1}, c_{2}, c_{3}\right\} \text { and } S=\left\{s_{i} \mid i=0,1, \cdots, 6\right\}
$$

be the set of linguistic terms whose details has been shown in figure 1 .

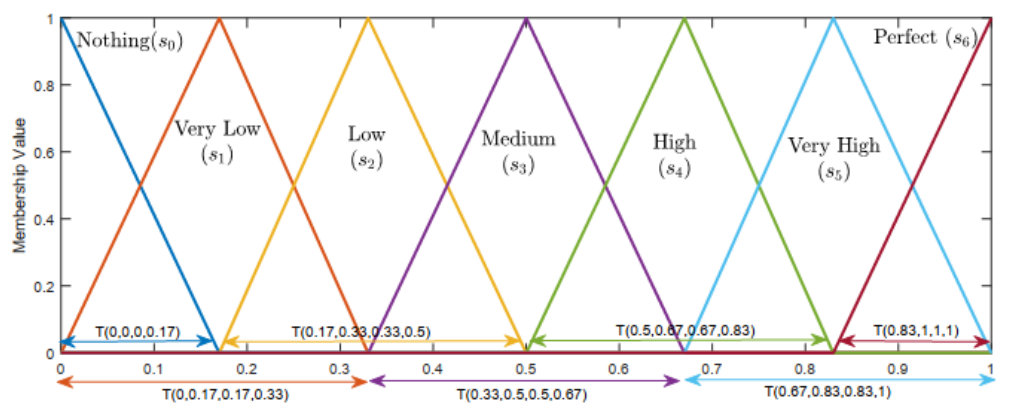

The assessment values for each alternative $x_{i}$ corresponding to each criterion $c_{j}$ are given the form of HFLM and its corresponding Fuzzy Envelopes are shown in table 1.

Table 1 Assessment Values of HFLM and its corresponding Fuzzy Envelopes

\begin{tabular}{|c|c|c|c|}
\hline$H_{S}^{i j}$ & $c_{1}$ & $c_{2}$ & $c_{3}$ \\
\hline \multirow{2}{*}{$x_{1}$} & $\{V L, L, M\}$ & $\{H, V H\}$ & $\{H\}$ \\
& $T(0,0.30,0.36,0.67)$ & $T(0.50,0.67,0.83,1)$ & $T(0.5,0.67,0.67,0.83)$ \\
\hline \multirow{2}{*}{$x_{2}$} & $\{L, M\}$ & $\{M\}$ & $\{N, V L, L\}$ \\
& $T(0.17,0.33,0.50,0.67)$ & $T(0.33,0.5,0.5,0.67)$ & $T(0,0,0.15,0.5)$ \\
\hline \multirow{2}{*}{$x_{3}$} & $\{H, V H, P\}$ & $\{V L, L\}$ & $\{H, V H, P\}$ \\
& $T(0.5,0.85,1,1)$ & $T(0,0.17,0.33,0.5)$ & $T(0.5,0.85,1,1)$ \\
\hline
\end{tabular}

After calculating Fuzzy envelops we aggregate it for each alternative $x_{i}$ according to the step 2 in section 4 .

$$
\begin{aligned}
& T\left(x_{1}\right)=T(0.33,0.55,0.62,0.83) \\
& T\left(x_{2}\right)=T(0.17,0.28,0.38,0.61) \\
& T\left(x_{3}\right)=T(0.33,0.62,0.78,0.83)
\end{aligned}
$$

Here we have taken $p=1$ for calculating distance between the different pair of alternatives. So, the preference relation matrix $P$ will be

$$
P=\left(\begin{array}{ccc}
N a N & 0.2225 & 0.0575 \\
0.2225 & N a N & 0.2800 \\
0.0575 & 0.2800 & N a N
\end{array}\right)
$$

The non-dominance degree for each alternative is

$$
\begin{aligned}
& N D D_{1}=\min \{1-0.2225,1-0.0575\}=0.775 \\
& N D D_{2}=0.7200 \\
& N D D_{3}=0.7200
\end{aligned}
$$

So, through our proposed system we have found that $1^{\text {st }}$ alternative i.e. $x_{1}$ is the best among the three alternatives, which is the same result of [7]. 


\subsection{Advantage}

The main advantage of the proposed expert system is to remove hesitant phase in group decision making process. The output of the system appears in Envelope form.

\section{Conclusion}

Most of the time expert provide their assessments by using single linguistic term. But in hesitant situation, experts needed to provide more precious linguistic expressions. Here HFLTS provide to increase flexibility in an indecisive case. For a decision-making system, an envelope for HFLTS is used as a linguistic interval, where as in the final result initial fuzziness loosed.

In this present study, the expert system is designed through the concept of fuzzy envelopes where the initial fuzzy representation of the linguistic terms is aggregate to fuzzy membership function without loss of the initial fuzziness. Invalidation processes using the same example and got the same result which has used in the decision-making model through the symbolic linguistic interval envelopes. But the advantage is that our output may not lose the initial fuzziness.

\section{References}

[1]. Bonissone P.P. (1980). A fuzzy sets based linguistic approach: theory and applications. Proceedings of the $12^{\text {th }}$ Conference on Winter Simulation.

[2]. Bordogna G. \& Pasi G. (1993). A fuzzy linguistic approach generalizing boolean information retrieval: A model and its Evaluation. Journal of the American Society for Information Science, 44:70- 82.

[3]. Kruskal J.B. (1964). Multidimensional scaling by optimizing goodness of fit to a nonmetric hypothesis. Psychometrika, 29(1): 1-27.

[4]. Ma J., Ruan D., Xu Y. \& Zhang G. (2007). A fuzzy-set approach to treat determinacy and consistency of linguistic terms in multi-criteria decision making. International Journal of Approximate Reasoning, 44:165-181.

[5]. Orlovsky S. (1978). Decision-making with a fuzzy preference relation. Fuzzy Sets and Systems, 1(3):155-167.

[6]. Pedrycz W. \& Song M. (2011). Analytic hierarchy process (AHP) in group decision making and its optimization with an allocation of information granularity. IEEE Transactions on Fuzzy Systems, 19:527-539 .

[7]. Rodriguez R.M., Martinez L. \& Herrera F. (2012). Hesitant fuzzy linguistic term sets for decision Making. IEEE Transactions on Fuzzy Systems, 20:109-119.

[8]. Ruspini E.H. (1969). A new approach to clustering. Information and Control, 15:22-32 .

[9]. Tang Y. \& Zheng J. (2006). Linguistic modelling based on semantic similarity relation among linguistic Labels. Fuzzy Sets and Systems, 157:1662-1673.

[10]. Yager R.R. (1988). On ordered weighted averaging aggregation operators in multicriteria decisionmaking. IEEE Transactions on Systems, Man, and Cybernetics, 18:183-190

[11]. Zadeh L.A. (1975). The concept of a linguistic variable and its application to approximate reasoning I. Information Sciences, 8:199-249. 